\title{
Integrating Molecular Mechanisms with Synaptic Plasticity in Neurological Disease
}

\author{
R. Nisticò • J. P. Bolaños • G. B. De Sarro
}

Received: 2 August 2012 /Accepted: 6 August 2012 /Published online: 26 August 2012

(C) Springer Science+Business Media, LLC 2012

Brain disorders are among the most disturbing and challenging pathologies in our time. A key lesson reinforced in the last two decades is that the processes that contribute to neurological disease are not exclusive to each disorder; rather, there are several common mechanisms. Recent studies have started to develop a molecular framework supporting the idea that alterations in normal synaptic function are not only a core feature but also a leading cause of disease. In this issue, Molecular Neurobiology decided to publish a set of reviews summarizing the current state of knowledge on the molecular and synaptic mechanisms of neurodegeneration that were discussed in a recent workshop on "Metabolic Requirements and Changes in Cell Proliferation and Death" held in Calabria, Italy.

One of the most challenging hypothesis explaining the molecular bases of neuronal apoptotic death in neurological disorders is the notion that neurodegeneration may be an aberrant attempt of postmitotic neurons to acquire a mitotic phenotype and reenter the cell cycle. In this special issue of Molecular Neurobiology, Almeida [1] revisits and provides potential mechanistic insights into the widely held notion that postmitotic neurons cannot divide. She focuses and describes how the regulation of a cell cycle-related E3 ubiquitin ligase - the anaphase promoting complex — keeps

R. Nisticò $(\bowtie)$

Laboratory of Experimental Neurology,

IRCCS Santa Lucia Foundation,

00143 Rome, Italy

e-mail: r.nistico@med.uniroma2.it

J. P. Bolaños

Department of Biochemistry and Molecular Biology,

University of Salamanca,

37007 Salamanca, Spain

G. B. De Sarro

Science of Health Department, School of Medicine and Surgery, School of Pharmacy, University "Magna Graecia" of Catanzaro, 88100 Catanzaro, Italy in check, by actively inducing protein destabilization, cyclin B1, and the glycolytic-promoting enzyme PFKFB3. This process strongly contributes to the typical neuronal postmitotic phenotype and survival, hence supporting the idea that novel therapeutic strategies should also be focused on modulating cell cycle-related targets. The above-mentioned lack of neuronal ability to divide may be overcome by replacing damaged neurons or by restoring their function. Thus, Kittappa et al. [2] revisit the molecular mechanisms responsible for neuronal renewal from stem cells, which are present in specific niches within the adult brain. Furthermore, the authors bring the novel notion that even non-terminally differentiated neural stem cells play roles in the regeneration of neurons and their synaptic function by mechanisms beyond mere cell replacement. These cells signal specific survival pathways that are worth investigating in our search for novel therapeutic strategies against neurodegeneration. According to this notion, noninvasive tools to follow-up synaptic function in the living brain are, therefore, essential for our better understanding of neuronal regeneration. In this context, Pilato et al. [3] discuss in detail the technical bases, as well as the clinical and experimental applications, of these new neurophysiological tools. The implementation of these techniques in routine research and clinical settings will not only provide us with a follow-up of the functional recovery of neurons but also information about how to treat drug-resistant neurological and psychiatric diseases.

These articles therefore highlight the notion that synapse loss and synaptic dysfunction are critical parameters to be evaluated during the setting or progress of neurodegenerative diseases; among these, Alzheimer's disease (AD) is the one affecting most people worldwide. In good agreement with the health and social importance of this disorder, three of the review articles of this special issue deal with different-but otherwise interconnected - aspects of the molecular mechanisms involved in AD. As Nisticò et al. [4] pinpoints in this special issue of Molecular Neurobiology, both synapse loss 
and synaptic dysfunction are highly correlated with the cognitive decline in AD. Evaluating the efficacy of novel therapeutic strategies by electrophysiological studies, such as hippocampal synaptic transmission and long-term potentiation analyses, remains an important task in the field. Careful should, however, be taken when translating preclinical studies into human trials. Nisticò et al. have thus highlighted the intrinsic limitations in the use of experimental systems and related challenges. Nevertheless, without yet a clear notion explaining the onset of $\mathrm{AD}$, groundbreaking novel aspects explaining the molecular mechanisms involved will surely provide new potential therapeutic targets against this disease. In this context, two further review articles highlight the importance of growth factors in the initial steps of the disease. Cattaneo and Calissano [5] review the notion that deficits in the signaling axis, nerve growth factor-TrkA receptor, would be an upstream driver in $\mathrm{AD}$ pathology by leading to abnormal processing of the amyloid- $\beta$ precursor protein and tau. In contrast, Giuffrida et al. [6] propose a challenging mechanism in which the deficiency of neuroprotective $\beta$-amyloid monomer protein would be responsible for the loss of pro-survival insulin-insulin growth factor-1 axis, rather than a downregulation triggered by the toxic $\beta$-amyloid oligomers. Thus, the cognitive decline in this devastating disease would be initiated by $\beta$-amyloid monomers deficiency, which takes place in the preclinical phase of $\mathrm{AD}$.

Inflammation appears to be in the underlying mechanism (s) triggering not only AD but also Parkinson's disease (PD), as well as amyotrophic lateral sclerosis. De Chiara et al. [7] herein bring the notion that chronic bacterial and viral infections would be behind the risk factors, according to increasing evidence from epidemiologic and experimental data, of these disorders. Activation of inflammatory processes and host immune responses causing neuronal dysfunction and loss of viability are among the possible explanations, which would act in synergy with aging and genetic predisposition. At the subcellular level, one of the hallmarks observed in experimental models of these diseases is a deficient autophagic process. Of particular interest is the link between defective autophagy, oxidative stress, and PD pathogenesis. Indeed, Janda et al. [8] critically revisits and explains the apparent controversy in the role played by autophagy in $\mathrm{PD}$, in view that both impaired and upregulated autophagy has been observed to trigger dopaminergic loss. They particularly focus on the regulatory role of reactive oxygen species in autophagy, an exciting and timely issue that is currently under intense debate.

Non-neurodegenerative neurological disorders are also important, both as a health and as a social problem. In this regard, a better understanding of the molecular pathways involved in some type of epilepsies can have high impact in finding new and more efficient therapeutic strategies. In this special issue of Molecular Neurobiology, Russo et al. [9] describes the tight involvement of an alteration in mammalian (mechanistic) target of rapamycin (mTOR) signaling pathway in this, and other neurological diseases, due to its pivotal role in apparently disparate functions such as cell proliferation and synaptic plasticity. In particular, authors review the reported effects, in animal models of both genetic and acquired epilepsies, of modulators of the mTOR pathway focusing on their potential beneficial neuroprotective and antiepileptogenic effects, highlighting future directions for the clarification of the mechanisms involved in mTOR inhibition efficacy. Finally, the importance of the muscle and myogenesis in particular in neurological diseases is becoming increasingly apparent. Interestingly nitric oxide (NO), besides its roles in the central nervous system, has emerged as an important positive regulator of myogenesis. In particular, the occurrence of nitric oxide synthase in the sarcolemma, and its displacement observed in Duchenne muscular dystrophy, has given much interest in the knowledge of the molecular mechanisms regulating myogenesis by NO. In view of the physiological impact that neurological problems might have on chronic muscle injury, De Palma and Clementi [10] review this issue and the implications for possible therapies in muscular dystrophy, both at the preclinical and at the clinical level.

We hope you will find this special issue informative and stimulating.

\section{References}

1. Almeida A (2012) Regulation of APC/C-Cdh1 and its function in neuronal survival. Mol Neurobiol. doi:10.1007/s12035-012-8309-2

2. Kittappa R, Bornstein SR, Androutsellis-Theotokis A (2012) The role of eNSCs in neurodegenerative disease. Mol Neurobiol. doi:10.1007/s12035-012-8303-8

3. Pilato F., Profice P., Ranieri F., Capone F., Di Iorio R., Florio L., Di Lazzaro V. (2012) Synaptic plasticity in neurodegenerative diseases evaluated by in vivo neurophysiological techniques Mol Neurobiol. doi:10.1007/s12035-012-8302-9

4. Nisticò R., Pignatelli M., Piccinin S., Mercuri NB, CollingridgeGL. (2012) Targeting synaptic dysfunction in Alzheimer's disease therapy. Mol Neurobiol. doi:10.1007/s12035-012-8324-3

5. Cattaneo, Calissano. Nerve growth factor and Alzheimer's disease: new facts for an old hypothesis. Mol Neurobiol. doi:10.1007/ s12035-012-8310-9

6. Giuffrida, Tomasello, Caraci, Chiechio, Nicoletti, Copani. Betaamyloid monomer and insulin/IGF-1 signaling in Alzheimer's disease. Mol Neurobiol. doi:10.1007/s12035-012-8313-6

7. De Chiara G., Marcocci ME, Sgarbanti R, Civitelli L, Ripoli C, Piacentini R, Grassi C, Garaci E, Palamara AT. (2012) Infectious agents and neurodegeneration. Mol Neurobiol. doi:10.1007/ s12035-012-8320-7

8. Janda E., Isidoro C, Carresi C, Mollace V. (2012) Defective autophagy in Parkinson's disease: role of oxidative stress. Mol Neurobiol. doi:10.1007/s12035-012-8318-1

9. Russo E, Citraro R, Constanti A, De Sarro G (2012) The mTOR signaling pathway in the brain: focus on epilepsy and epileptogenesis. Mol Neurobiol. doi:10.1007/s12035-012-8314-5

10. De Palma C, Clementi E (2012) Nitric oxide in myogenesis and therapeutic muscle repair. Mol Neurobiol. doi:10.1007/s12035012-8311-8 